\title{
Obesity and childhood survivors of acute lymphoblastic leukemia: Do genetics play a role?
}

\author{
Cheryl Mele ${ }^{1,2^{*}}$ \\ ${ }^{1}$ Healthcare of Genetics Program, Clemson University, Clemson, USA \\ ${ }^{2}$ Drexel University, Philadelphia, USA \\ Email: cm3242@drexel.edu
}

Received 11 December 2011; revised 26 January 2012; accepted 9 February 2012

\begin{abstract}
Childhood survivors of acute lymphoblastic leukemia (ALL) are increased risk of several chronic complications, such as second cancers, pulmonary, metabolic complications and cardiovascular disease. Obesity and metabolic syndrome is one of the most common treatment related complication in children surviving cancer, which concurs with our nations childhood epidemic [1-3] Recent research has identified the role of genetics in the development of obesity and metabolic syndrome in childhood survivors of ALL. Growth hormone deficiency, Leptin regulation, fat mass obesity (FTO) gene and the insulin resistant ENPP1 variants disorders has been associated adverse effects of chemotherapeutic treatment and the cause of clinical manifestations of metabolic syndrome [4-8]. The illumination of the role of genetic variants can shed insights into obesity within high risk population, as well as, a target to prevent disease.
\end{abstract}

Keywords: Genetic Polymorphisms; Obesity; Metabolic Syndrome; Acute Lymphoblastic Leukemia

\section{INTRODUCTION}

Acute lymphoblastic leukemia is the most common form of childhood cancer with an $80 \%$ cure rate [9]. As long term survivors, it is important to address health care risks and long term effects of their treatments. A number of endocrine/metabolic adverse effects have been described in the literature in childhood survivors of ALL, which include obesity, dyslipidemia, insulin resistance, hypertension and eventually cardiac disease [2,3,5]. The high rate of obesity $\left(\mathrm{BMI}>30 / \mathrm{kgm}^{2}\right)$ and metabolic syndrome has been reported in numerous studies, with a variance in prevalence $(11 \%-40 \%)$ dependent upon the method of measurement $[7,10-12]$. In large study of diverse popula-

*Drexel University Assistant Professor; Clemson University Doctoral Student in Healthcare of Genetics Program. tion in North American survivors of childhood ALL, who were a mean age of 32 years at follow-up, the studied reported not only an increased risk of obesity, but a significantly greater rate of change in BMI over time, as compared with a noncancer comparison population [13]. In an investigation by Razzouk, et al. (2007), overall percentage of survivors who were overweight or obese had approximated rates of prevalence as in the general population of the United States. However, children diagnosed at a younger age ( $<6$ years) and undergoing cranial radiation showed an increase risk for obesity and/or metabolic syndrome [14]. Overall, studies of childhood survivors showed an increase risk for obesity in metabolic syndrome especially due to particular treatment methods [7,10-14]. Many of ALL survivors are being followed in primary care settings and it's imperative that healthcare providers be familiar with consequences of treatment and offer evidence based lifestyle practices to patients and family.

\section{THERAPY IN ALL AND OBESITY/METABOLIC SYNDROME}

Survivors of pediatric ALL are increased risk of developing obesity; however, treatment related aspects and their role are not clear. Amongst ALL survivors, cranial radiation, chemotherapy agents, and steroids have been documented as a contributor [8]. Childhood survivors treated with cranial radiation, especially doses of $20 \mathrm{~Gy}$ or greater has an association with increased BMI/obesity $[10,11]$. Oeffinger and colleagues (2003) reported increased incidence of obesity, particularly in females receiving cranial radiation treatment especially at a young age $(<5 \mathrm{yr})$ [10]. It is hypothesized that cranial radiotherapy could be damaging to hormonal centers in the brain such as the leptin receptors which are critical for normal food intake and metabolism $[1,5,8]$. Other studies have shown that children treated with cranial radiation are more susceptible to growth hormone deficiency [5, $6,8,12]$. Growth hormone deficiency is associated with 
obesity and increased abdominal circumference, hyperlipidemia, hypertension which are symptoms of metabolic syndrome [7].

In contrast, Razzouk et al. study (2007), compared BMI with high dose treatment intrathecal mehtotrexate, and various doses of cranial radiation. [14]. They found higher doses of Gy in males, and older male children developed a higher BMI but the change over time was insignificant [14]. Razzouk et al. (2007) concluded that other factors beside cranial radiation contributed to increase risk of obesity in ALL [14]. Other mechanism which have been postulated to correlate with the development of obesity in survivors of ALL are poor dietary choices, changes in appetite regulation and decrease physical activity $[8,12]$. These factors also are found in the general population and confirm that obesity is a complex disease.

The specific reason for the weight gain during the chemotherapy is unknown but chemotherapeutic agents and notably corticosteroids play a major role. It is hypothesized the toxic effects from central nervous system agents such as intrathecal methotrexate or cytosine arabinoside is due to abnormal clearance and/or subsequent treatments [8]. However, the chemotherapeutic agents have felt to impose a lesser impact with the development of obesity than the glucocortcoid steroids [7]. Glucocortcoid steroids are part of a standard protocol for ALL therapy and when especially administered in long term cycles they are significant contributor to obesity in ALL [15] Glucocorticoid steroids cause significant weight gain, changes in body composition, and alterations in linear growth during the first months of treatment which continues through the first 2 years after therapy withdraw [8].

Body composition changes have been associated with premature adiposity rebound, which has a strong correlation with adult obesity [8]. The adiposity rebound (AR) normally occurs at $5-7$ years of age. Adipose rebound occurs early in ALL patients between the ages of $3-5$ years of age $[8,13,16]$. Obesity-related health risk is greatest in those with an abdominal obesity phenotype and specifically, excess accumulation of visceral fat within the abdomen is strongly associated with morbidity and mortality $[8,16]$. Emerging evidence also suggests that the storage of fat in non-adipose tissues such as liver and skeletal muscle may carry an independent health risk as well [16]. The early adipose rebound is thought to be a consequence of treatment with cranial radiation and, glucocorticoid steroids which alters body composition and increased risk of developing obesity/metabolic syndrome $[8,16]$. A similar AR situation is seen in IUGR, low birth weight and hypothyroidism where as early rebound resulted drastic changes in body composition, insulin resistance and metabolic syndrome later in life [8].

It has been increasingly acknowledged that obesity in humans is a multifaceted disorder and survivors of ALL are at increased risk. Many of the aforementioned studies postulate that development of obesity after treatment of ALL depends upon a number of factors such as genetic background, acute and chronic conditions and environmental interaction. Table 1 summarizes specific mechanisms associated with the promotion of obesity and metabolic syndrome in childhood survivors of ALL.

\section{GENETIC POLYMORPHISMS AND ALL}

Reports in the literature have identified many genetic polymorphisms associated with obesity and metabolic syndrome in children. Leptin, a hormone encoded by the OB gene (obese gene mapped on chromosome 7), and secreted mainly by adipose tissue acts as a satiety signal in a feedback loop with the hypothalamic centers in the brain which controls feeding behaviors, appetite, energy expenditures and temperature regulation [15,17]. Simply, leptin is a signal from the adipose tissue that tells the brain about the size of the fat mass [17]. Genetic alteration or deficiency in leptin or its receptors provokes hyperphagia, obesity and, insulin resistance [17]. Ross and fellow researchers explored the potential association between the Gln223 Arg polymorphism in the leptor gene and the mounting risk of obesity [17]. Also, several investigations and particularly one from the Cancer Survivor Study, found genetic variation in the leptin receptor gene influenced the development of obesity, especially in female survivors who experienced cranial radiation. [6, 10,17-20]. This disruption in leptin regulation and leptin resistance in children with ALL has correlated positively with an elevated BMI during and after treatment $[7,8,16]$. Moreover, leptin deregulation is known to be a part of a function in leukemia physiopathology as its receptors, which are participants of the cytokine super family and are expressed in leukemia blast cells $[8,16,17]$. It is noteworthy that the pathways of metabolic regulation are

Table 1. Identify mechanism for obesity and metabolic syndrome in ALL survivors.

\begin{tabular}{|c|c|}
\hline ALL Treatments & Other Mechanisms \\
\hline Cranial Radiation (12 - 24 Gy) & Genetic alterations \\
\hline Leptin gene alterations & Familial background \\
\hline Premature Adipose Rebound & Dietary choices \\
\hline $\begin{array}{l}\text { Growth hormone deficiency due to Cranial } \\
\text { radiation }\end{array}$ & Reduced physical activity \\
\hline Chemotherapy agents (<common) & \\
\hline $\begin{array}{l}\text { Sivero-Miachon et al. "Adiposity in } \\
\text { Childhood Cancer Survivors: Insights into } \\
\text { Obesity Physiopathology." Arq Bras } \\
\text { Edocrinol Metab., 53, 2, 2009, 190-199 }\end{array}$ & \\
\hline
\end{tabular}


conducted by the hypothalamus and that cranial radiation causes disorder [17]. Thus, the hypothalamus is one of the most important regions affected by treatment of ALL. Insulin and leptin recaptors located in key regions of the hypothalamus arcuate nucleus are impaired by cranial radiation and chemotherapy (macrophage/inflammatory markers) which results in leptin resistance [17]. Alterations in the leptin/insulin feedback mechanisms in turn may explain the high incidence of obesity/metabolic syndrome in ALL survivors [8,17].

An association of single nucleotide polymorphisms (SNPs) in the fat mass and obesity-associated (FTO) gene region was identified in multiple populations and involved in the pathogenesis of obesity and metabolic syndrome [21,22]. The FTO gene is composed of nine exons and located on chromosome 16 [21,22]. Studies in rodents have demonstrated that FTO gene is abundant in the hypothalamus of the brain and regulates energy balance [21-23]. Variations in the FTO gene has been associated with increase weight, body mass index (BMI), and fat mass, and early onset obesity [21-23]. It has been suggested by scientist that a significant association between the rs9939609, an allele variant was responsible for higher incidence of obesity in patients after ALL treatment and associated with 31\% increase of developing obesity $[8,18,21]$. Because the FTO gene is associated with increase fat mass and altered energy expenditure, it correlates with the increased serum leptin levels and control of energy homeostasis [8,18,21-23]. However, the effects of the FTO genetic polymorphisms and ALL childhood survivors have not been studied extensively as the leptin genetic polymorphisms.

Survivors of acute lymphoblastic leukemia (ALL) are at increased risks of impaired glucose metabolism, insulin resistance, and metabolic syndrome with known genetic risks [24] It is inferred that genetic polymorphisms affecting B-cell function and alterations in glucose metabolism (PAX4) and transcription factor 7-like 2 (TCF7L2) play a critical role in pancreatic B-cell development and/or function [25,26] Moreover, mutations or polymorphisms that weaken transcriptional behaviors would contribute to reduction in B-cell survival and/or proliferation during the ALL treatment [23]. Thus, subsequent gradual loss of insulin producing cells [24] Surapolchai et al. (2009), found genetic polymorphisms in the PAX4 R192H gene was nominally associated with impaired glucose tolerance [24]. Furthermore, the allele frequency of polymorphisms in the PAX4 and TCF7L2 gene in ALL survivors were not different from non-diabetic controls, and Surapolchai et al. (2009), concluded that insulin resistance in survivors could not be explained by genetic susceptibility to diabetes but probably associated with gene-drug interaction that influences the B-cell survival after chemotherapy administration [24]. Conse- quentially, further longitudinal research and longer follow-up care is needed to gain better perception of the etiology and influencing factors of developing alterations in glucose metabolism and metabolic syndrome in childhood survivors of ALL [23]. The clinical and genetic predictors of altered glucose metabolism could be identified early on in treatment of ALL, aiming to identify and monitor vulnerable groups of survivors.

\section{IMPLICATIONS FOR HEALTHCARE PROVIDERS}

Obesity and metabolic syndrome although a common complication for childhood survivors of ALL, the related causes are complex. The prevalence and pathogenesis of developing obesity and metabolic syndrome in survivors and the relationship between multiple factors has not been clarified. Further research should focus on the understanding of the pathophysiological basis of developing the disorder, genetic polymorphisms and lifestyle alterations. There is evidence that genetic variations with adipose dysfunction and insulin resistance could play a role in the development of obesity in cancer survivors. Interesting to note a study by Yun et al. (2010) demonstrated that obesity in children with ALL can accelerate the disease. Therefore, it is important to identify any genetic risk and provide early interventions in order not to compromise a child's recovery [27].

Studies have further suggested early identification and intervention strategies during maintenance phase of ALL treatment is a feasible option in order to prevent the development of obesity and metabolic syndrome in childhood survivors of ALL [28,29]. Many childhood ALL studies have demonstrated that a high proportion of children show rapid and excessive weight gain soon after diagnosis, partially due to decrease activity and treatments $[30,31]$. In addition, recent study indicated that dietary intake for many adult survivors of childhood ALL is not concordant with dietary recommendations that reduce their risk of obesity and cardiovascular disease [32].

In conclusion it may be possible to lower rates of obesity and metabolic syndrome by early identification of high risk groups and followed by timely interventional evidence based plan. A prospective early research design could include identification of genetic polymorphisms such as leptin receptor genes, FTO genes and insulin receptor genes in correlation with body mass changes in the early phases of treatment for ALL. Since children with ALL have early adipose rebound, and excessive visceral and abdominal fat, perhaps abdominal circumference measurements should be incorporated in the standard anthropometric measurements monitored during primary care visits. Such measures can alert providers to early risk and promote timely interventional treatments. 
Also, integrating nutritional and exercise counseling for the patient and families in the initial phase of treatment and throughout follow-up in the primary care setting is also an essential component.

\section{REFERENCES}

[1] Karman, S. et al. (2010) Late effects of childhood ALL treatment on body mass index and serum leptin levels. Journal of Pediatric Endocrinology Metabolism, 23, 669674. doi:10.1515/JPEM.2010.23.7.669

[2] Pakakasama, S. et al. (2010) Late effects of childhood acute lymphoblastic leukemia: A study from the Thai pediatric oncology group. International Journal of Hematology, 91, 785-854. doi:10.1007/s12185-010-0594-9

[3] Tabon, M. and Leverger G. (2009) Outcome of children cured of Acute lymphoblastic Leukemia. Bulletin de l'Académie Nationale de Médecine, 193, 1519-1528.

[4] Bottcher, Y. et al. (2006) ENPP1 Variants and haplotypes predispose to early onset obesity and impaired glucose and insulin metabolism in german obese children. Journal of Clinical Endocrinology and Metabolism, 91, 49484952. doi: $10.1210 /$ jc.2006-0540

[5] Kourti, M. et al. (2005) Metabolic syndrome in children and adolescents with acute lymphoblastic leukemia after completion of chemotherapy. Clinical and Laboratory Observations, 27, 499-501.

[6] Ross, J. (2006) Genetic susceptibility and body mass in childhood cancer survivors. Pediatric Blood Cancer, 48, 731-735. doi:10.1002/pbc.21112

[7] Sivero-Miachon, A. et al. (2008) Detection of metabolic syndrome features among childhood cancer survivors: A target to prevent disease. Vascular Health and Risk Management, 4, 825-836.

[8] Siviero-Miachon, A., Spinla-Castro, A. and Guerra-Junior G. (2009) Adiposity in childhood cancer survivors: Insights into obesity physiopathology. Arquivos Brasileiros de Endocrinologia e Metabologia, 53, 190-199. doi:10.1590/S0004-27302009000200011

[9] Pui, C.H. et al. (2003) Results of therapy for acute lymphoblastic leukemia in black and white children. JAMA 290, 2001-2007. doi:10.1001/jama.290.15.2001

[10] Oeffinger, K.C. et al. (2003) Obesity in adult survivors of childhood acute lymphoblastic leukemia: A report from the childhood cancer survivor study. Journal of Clinical Oncology, 21, 1359-1365. doi:10.1200/JCO.2003.06.131

[11] Rogers, P.C. et al. (2005) Obesity in pediatric oncology. Pediatric Blood Cancer, 45, 881-891. doi: $10.1002 / \mathrm{pbc} .20451$

[12] Nathan, P.C. et al. (2006) The prevalence of overweight and obesity in pediatrics survivors of cancer. Journal of Pediatric, 149, 518-525. doi:10.1016/i.jpeds.2006.06.039

[13] Garmey, E. et al. (2008) Longitudinal changes in obesity and body mass index amon adult survivors of childhood acute lymphoblastic leukemia: A report From the childhood cancer survivor study. Journal of Clinical Oncology, 26, 4639-4645. doi:10.1200/JCO.2008.16.3527
[14] Razzouk, B. et al. (2007) Obesity in childhood survivors of childhood acute lymphoblastic leukemia and lymphoma. Journal of Clinical Oncology, 5, 1183-1189. doi:10.1200/JCO.2006.07.8709

[15] Armenian, S. and Bhatia, S. (2009) Chronic health conditions in childhood cancer survivors: Is it ALL treatment related or do genetics play a role. Journal of General Internal Medicine, 24, 395-400. doi:10.1007/s11606-009-0995-8

[16] Janiszewski, P.M., et al. (2007) Abdominal obesity, liver fat, and muscle composition in childhood survivors of acute lymphoblastic leukemia. Journal of Clinical Endocrinology Metabolism, 92, 3816-3821. doi:10.1210/jc.2006-2178

[17] Hino, M., Nakao, T., Yamane, T. (2000) Leptin receptor and leukemia. Leukemia Lymphoma, 35, 457-461. doi:10.3109/10428190009148392

[18] Skoczen, S., Surmiak, M. and Wojciech, S. (2010) Survivors of acute lymphoblastic leukemia and body mass changes. Expert Opinion Drug Safety, 9, 65-76. doi: $10.1517 / 14740330903410213$

[19] Ross, J. and Oeffinger, K.C. (2004) Genetic variation in the leptin receptor gene and obesity in survivors of childhood acute lymphoblastic leukemia: A report from the childhood cancer survivor study. Journal of Clinical Oncology, 22, 3558-3562. doi:10.1200/JCO.2004.11.152

[20] Van Waas, M. and Neggers, S.J.C.M.M. (2010) Components of the metabolic syndrome in 500 adult long-term survivors of childhood cancer. Annals of Oncology, 21, 1121-1126. doi:10.1093/annonc/mdp414

[21] Fawcett, K. and Barroso I. (2010) The Genetics of obesity: The FTO gene leads the way. Trends Genetics, 26, 266274. doi:10.1016/j.tig.2010.02.006

[22] Rendo, T. et al. (2009) Effects of the FTO gene on lifestyle interventions in children. Obesity Facts, 2, 393-399. doi:10.1159/000262296

[23] Wang, T. et al. (2010) The association between common genetic variation in the FTO gene an metabolic syndrome in Han Chinese. Chinese Medical Journal, 123, 18521858.

[24] Surapolchai, S. et al. (2010) Impaired glucose tolerance and insulin resistance in survivors of childhood acute lymphoblastic leukemia: Prevalence and risk factors. Journal of Pediatric Hematology Oncology, 32, 383-389.

[25] Helgason, A. et al. (2007) Refining the impact of TCF7L2 gene variants on type 2 diabetes and adaptive evolution. Nature Genetics, 39, 218-225.

[26] Plengvidhya, N. et al. (2007) PAX4 mutations in thais with maturity onset diabetes of the young. The Journal of Clinical Endocrinology \& Metabolism, 92, 2821-2826. doi:10.1210/jc.2006-1927

[27] Yun, J.P. et al. (2010) Diet-induced obesity accelerates acute lymphoblastic leukemia progression in two murine models. Cancer Prevention Research, 3, 1259-1264. doi:10.1158/1940-6207.CAPR-10-0087

[28] Collins, L. et al. (2009) Growth in children with acute lymphoblastic leukemia during treatment. Journal of Pediatric Hematology Oncology, 32, 304-307. 
doi:10.1097/MPH.0b013e3181ece2bb

[29] Withcombe, J. et al., (2009) Weight patterns in children with higher risk of ALL: A report from the Children's Oncology Group (CCG) for CCG 1961. Pediatric Blood Cancer, 53, 1249-1254.

[30] Jansen, H. et al. (2009) Acute lymphoblastic leukemia and obesity: Increased energy intake or decreased physical activity? Support Cancer Care, 17, 103-106. doi:10.1007/s00520-008-0531-0
[31] Reilly, J. (2009) Obesity during and after treatment for childhood cancer. Endocrine Development, 15, 40-58. doi:10.1159/000207609

[32] Robien, K. et al. (2008) Poor adherence to dietary guidelines among adult survivors of childhood acute lymphoblastic leukemia. Journal of Pediatric Hematology Oncology, 11, 815-822.

doi:10.1097/MPH.0b013e31817e4ad9 